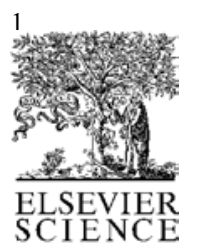

Submitted to Elsevier Science

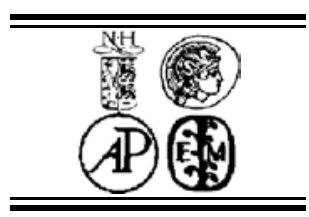

\title{
Loss and Gain in Bloch Oscillating Super-Superlattices: THz Stark Ladder Spectroscopy
}

\author{
Peter Robrish $^{\mathrm{a}}$, Jing Xu ${ }^{\mathrm{b}}$, Shigeki Kobayashi, ${ }^{\mathrm{c}, \mathrm{d}}$, Pavlos G. Savvidis ${ }^{\mathrm{c}}$, Borys Kolasa ${ }^{\mathrm{b}}$, \\ Greg Lee ${ }^{\mathrm{a}}$, Dan Mars ${ }^{\mathrm{a}}$ and S. James Allen ${ }^{\mathrm{b}, \mathrm{c}}$ \\ ${ }^{a}$ Agilent Laboratories, 3500 Deer Creek Rd.,Palo Alto, CA 94304 , USA \\ ${ }^{b}$ UCSB,Physics Dept., Santa Barbara, CA 93106, USA \\ ${ }^{c} U C S B$, Institute for Quantum and Complex Dynamics, Santa Barbara, CA 93106, USA \\ ${ }^{d}$ The University of Tokyo, Institute of Industrial Science,4-6-1 Komaba, Meguro-ku,Tokyo 153-8505, Japan
}

Elsevier use only: Received date here; revised date here; accepted date here

\begin{abstract}
Bloch oscillation in electrically biased semiconductor superlattices offer broadband terahertz gain from DC up to the Bloch frequency or Stark splitting. Useful gain up to 2-3 terahertz can provide a basis for solid-state electronic oscillators operating at 10 times the frequency of existing devices.

A major stumbling block is the inherent instability of the electrically biased doped superlattices to the formation of static or dynamic electric field domains. To circumvent this, we have fabricated super-superlattices in which a large superlattice is punctuated with heavily doped regions. The short superlattice sections have subcritical " $n l$ " products

Room temperature, terahertz photon assisted transport in short InGaAs/InAlAs superlattice cells allows us to determine the Stark ladder splitting as the superlattice is electrically biased and confirms the absence of electric field domains in short structures.

Absorption of radiation from 1.5 to $2.5 \mathrm{THz}$ by electrically biased InAs/AlSb super-superlattices exhibit a crossover from loss to gain as the Stark ladder is opened. Measurements are carried out at room temperature in a novel planar terahertz waveguide defined by photonic band gap sidewalls and loaded with an array of electrically biased super-superlattices. The frequency dependent crossover voltage indicates $\sim 80 \%$ participation of the super-superlattice.
\end{abstract}

(C) 2001 Elsevier Science. All rights reserved

PACS: 73.21.Cd; 74.78.Fk;

Keywords: Semiconductor superlattice; Terahertz; Bloch oscillator; Photon assisted transport. 


\section{Introduction}

The terahertz part of the electromagnetic spectrum is marked by a lack of commercial technology. This is readily apparent if we focus on fundamental solidstate oscillators or sources, Fig. 1. On the electronic end the technology drive has pushed quantum transport devices like resonant tunneling diodes but the most effective solid state sources of terahertz radiation are harmonic generators [1]. On the photonic end the most impressive inroads are made by the development of long wavelength quantum cascade lasers (QCL's), [2,3].

Bloch oscillating superlattices have the potential to provide broad band gain at terahertz frequencies and may be the basis of a technology that will help fill the gap in solid state terahertz fundamental oscillators. The seminal work of Esaki and Tsu [4]
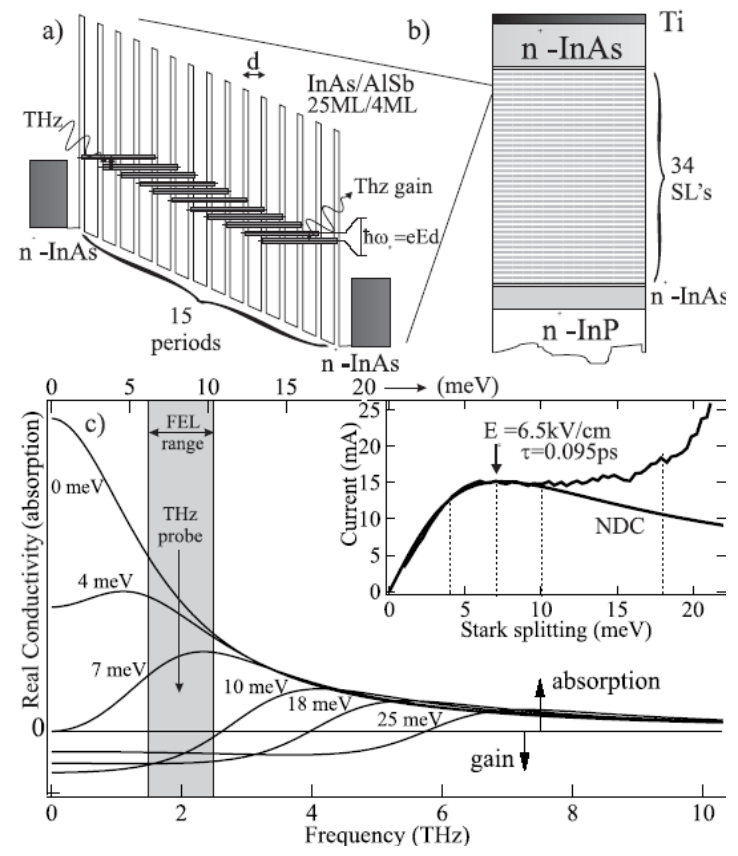

Fig. 2 (a) A superlattice structure under electrical bias. (b) A stack of short superlattice cells. Lower: The differential conductance is expected to be suppressed and exhibit negative conductance up to the Bloch frequency of Stark ladder rung spacing. From Ref. 6.

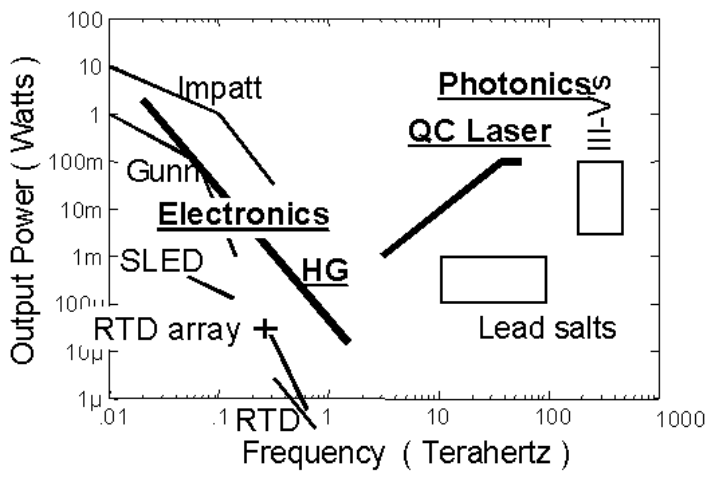

Fig. 1 The terahertz technology landscape. The low frequency end of the terahertz spectrum engages electronic transport devices; the high frequency end photonic quantum transition devices.

drew attention to the fact that the relatively large scale periodic structures in semiconductor superlattices made them ideal solids in which to explore and use Bloch oscillation. Following this work that predicted that electrically biased semiconductor superlattices will exhibit negative differential conductivity (NDC) [4] like the Gunn effect, Ktitorov et al showed theoretically that the negative resistance and potential gain should persists up the Bloch frequency or spacing between the rungs of the Stark ladder [5]. Unlike the Gunn effect which persists only up to the energy relaxation rate, 100-200 $\mathrm{GHz}$, electrically biased semiconductor superlattices should exhibit gain over a much larger band width, up to several terahertz. See Fig. 2. [6]

It is clear that we should set aside naive models of a Bloch oscillator device. A Bloch oscillator device does not oscillate at the Bloch frequency, it oscillates at a frequency determined by the external resonator or circuit but only at frequencies below the Bloch frequency.

\section{Stabilizing the electric field.}

It is well known that a medium with bulk negative differential conductivity (NDC) exhibits space charge instabilities, which lead to electric field domains and current self-oscillations [7,8]. But, if the material is sufficiently short, small space charge fluctuations that could grow and provide electric field domain walls are swept out before a domain wall is established. As 
in the Gunn effect, we can define a critical " $\mathrm{nL}$ " product below which propagating electric field domains are suppressed. Here, $\mathrm{n}$ is the electron density and $\mathrm{L}$ is the sample length. if

For a semiconductor superlattice we estimate that

$$
n L<7 \varepsilon \frac{E_{c}}{e},
$$

where $\varepsilon$ is the dielectric constant and $E_{c}$ the critical electric field at which NDC sets in, space charge instabilities are swept out of the superlattice before they form a domain wall $[9,10]$. For superlattice doping densities of the order of $2.5-5.010^{16} \mathrm{~cm}^{-3}$, and critical fields of the order of $\sim 6 \mathrm{kV} / \mathrm{cm}$, critical lengths are of the order of 100-200 nm. For superlattice periods of $10 \mathrm{~nm}$ this requires lengths less than 10-20 quantum wells, very short superlattices indeed.

On the other hand to mitigate the effects of parasitic contact resistance, shunt capacitance and to ultimately deliver useful power, long superlattices, of the order of 5-10 $\mu \mathrm{m}$ are needed. To satisfy both requirements, short superlattices and long devices, we fabricate super-superlattices comprised of a stack of short superlattices, each short section separated from its neighbor by a heavily doped region. (Fig. 2)

\section{Stark ladder spectroscopy.}

To experimentally confirm the notion that short superlattice sections will sustain a stable and reasonably uniform electric field, terahertz photon assisted transport measurements were performed on various test structures. These consisted of short superlattices (cells) of either 9 or 15 quantum wells sandwiched between $\mathrm{n}^{+}$regions. Samples that contained stacks of 1,2 or 5 of these cells were tested. 18 or $14 \mu \mathrm{m}$ diameter mesas were etched and the chip mounted and bonded at the end of an SMA connector. Special electronics measured the currentvoltage (I-V) characteristic of the device under test (DUT) on a time scale of the order of $\sim 1 \mu \mathrm{sec}$. In this manner the entire I-V could be measured while the several microsecond long terahertz radiation from the UCSB free-electron lasers was focused on the device.

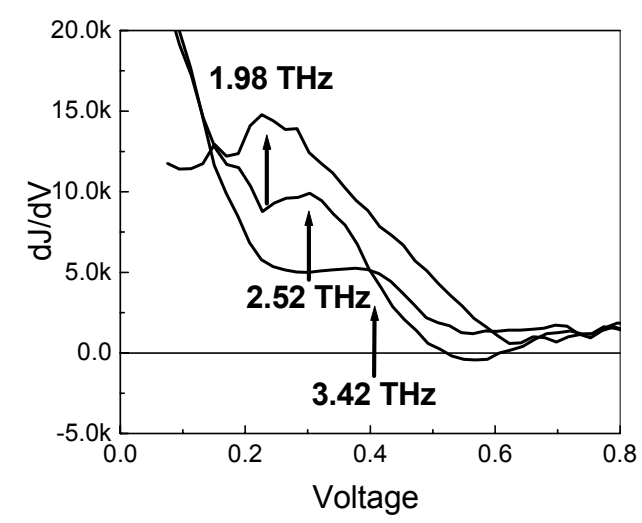

Fig. 3 The terahertz irradiated differential conductance, $d J / d V$, of a 2 cell stack. Each cell consists of a 15 quantum well, InGaAs/InAlAs, superlattice. A peak in differential conductance appears at a voltage that depends on frequency.

Fig. 3 shows the differential conductance, $d J / d V$, of a terahertz irradiated 2 cell stack. Here each cell consists of a 15 quantum well, InGaAs/InAlAs, superlattice. A peak in differential conductance appears at a voltage that depends on frequency. In essence, when the spacing of the rungs of the Stark ladder exceed the terahertz photon energy the electrons are driven down the ladder, causing an increase in current (positive $d J / d V$ ), feeding energy into the radiation field (the gain channel). [11,12]

A quantitative description of this phenomenon follows much earlier developments of microwave photon assisted transport in superconducting

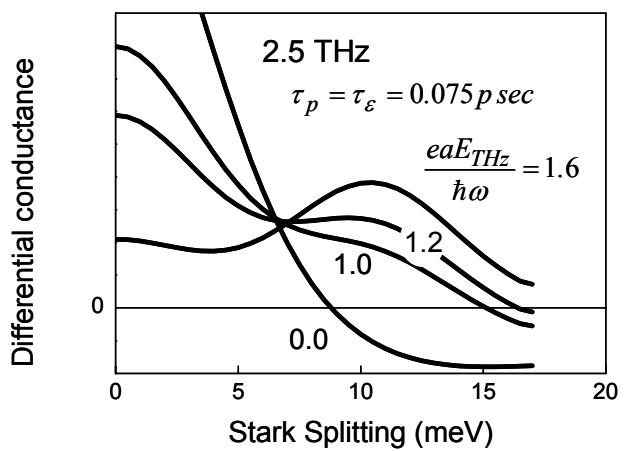

Fig. 4 Model calculation of differential conductance with terahertz field strength as a parameter. 
junctions. [13] The DC current flow in the presence of a DC voltage and strong terahertz field may be expressed as [14]

$$
I(V)=\sum_{n=-\infty}^{\infty} J_{n}^{2}\left(e a E_{T H z} / \hbar \omega\right) \cdot I_{0}(V+n \hbar \omega / e)
$$

where $V$ is the applied DC voltage, $I$, the current, and $I_{0}$ the current in the absence of terahertz radiation. The superlattice period is $a$, the strength of the terahertz field is $E_{T H z}$ at frequency $\omega . \quad \hbar$ and $e$ are Planck's constant and the electron charge. Fig. 4 and Fig. 3 may be compared, extracting an estimate of the internal terahertz field strength. More important the comparisons quantitatively support the notion that the

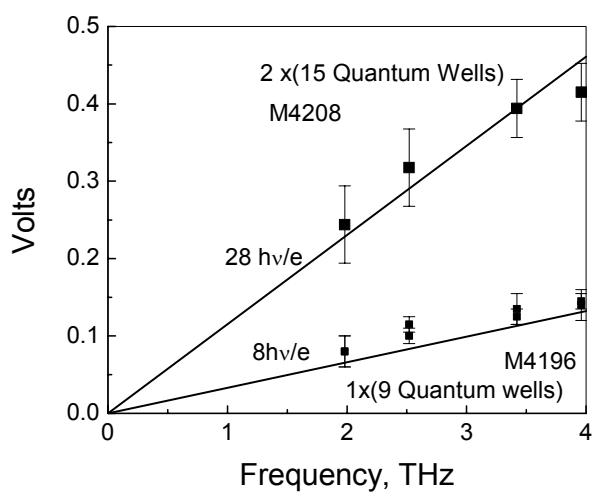

Fig. 5 Voltage bias at differential conductance maxima vs. terahertz frequency. Upper data: 1 stacked 15 quantum well cells. Lower data: a single cell of 9 quantum wells.

peak in differential conductance corresponds to resonance of the terahertz radiation and Stark ladder rung spacing.

Fig. 5 exhibits the voltage of the differential conductance maxima vs. terahertz frequency. The solid lines correspond to the expected voltage vs. frequency if the voltage were uniformly dropped across the superlattice sections. We note here that there are $(n-1)$ separations for $n$ quantum wells in a single cell.

\section{Loss and Gain under Electrical Bias}

Experimentally, photon assisted transport proceeds in a relatively straightforward manner; the sample is the detector. Details concerning coupling of the terahertz radiation to the sample are not important provide that enough power is available and the desired changes in differential conductance appear.

Measurement of the dynamical conductance, which is directly related to "loss and gain" in electrically biased superlattices, requires more finesse. Here, we load a terahertz waveguide with a row of super-superlattices and measure the transmission through the waveguide as a function of electrical bias.

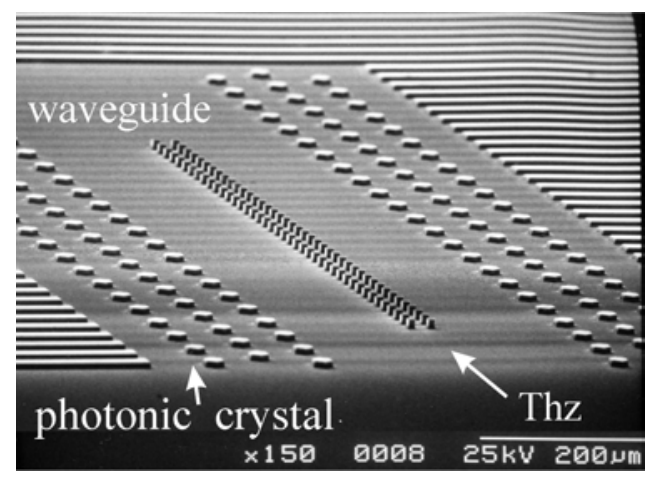

Fig. 6 Terahertz waveguide with metallic top removed. The sidewalls are defined by a photonic bandgap structure. Radiation enters the $10 \mu \mathrm{m}$ high waveguide and exits through a hole in the metallic top and is directed to a detector.

The wave guide is formed by etching the superlattice wafer as shown in Fig. 6. The $\mathrm{n}^{+}$ substrate forms the bottom of the waveguide and metal, gold plated brass, the top. A hole in the top of

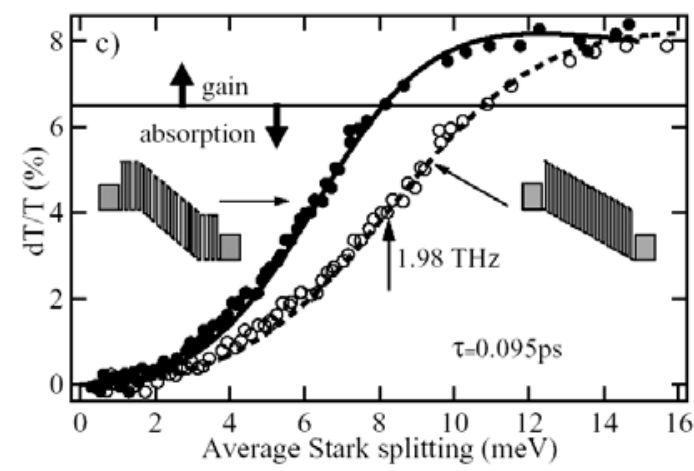

Fig. 7 Fractional change in transmission vs. applied voltage expressed as average Stark ladder splitting. Solid points are the data; open circles expected voltage dependence. 
the guide directs the transmitted terahertz radiation to a detector. Transmission is measured as a function of electrical bias applied to the row of super-superlattice mesas in the center of the guide.

Transmission measurements were carried out at frequencies between 1.5 and $2.5 \mathrm{THz}$ (Fig. 2). Fig. 7 shows the change in transmission vs. voltage at 1.98 THz. [6] The voltage required to open the transmission through the guide is about $80 \%$ less than expected which implies that $\sim 20 \%$ of the superlattice experience little or no field. This result is obtained at each frequency between 1.5 and $2.5 \mathrm{THz}$. The voltage required to open the transmission is proportional to frequency but $\sim 75-80 \%$ of that expected.

Unlike the test structures used for the Stark ladder spectroscopy, here there was no "set back" region between the $\mathrm{n}^{+}$and short superlattice. Spill over from the heavily doped region may effectively "short-circuit" several quantum wells at the edges of the superlattice.

\section{Conclusions}

Stark ladder spectroscopy by terahertz photon assisted transport and terahertz waveguide measurements of the bias dependent dynamical conductance, indicate that short period superlattices can be used to achieve essentially uniform electric fields. While the field distribution of sub critical bulk negative resistance material should have no static or dynamic domains, the field is not expected to be entirely uniform. But the gain bandwidth of these materials is expected to be very broad (Fig. 2) and modest slowly varying electric fields will be of little consequence. But the Stark ladder spectroscopy directly speaks to this issue and it is apparent that for the range of fields and doping presented here the field distribution is quite uniform.

The immediate future indicates growth of appropriate super-superlattices, fabrication of various ring and ridge resonators and exploration of coherent terahertz emission.
Acknowledgements.

Work at UCSB was supported by the ARO, DARPA/ONR and CNID.

\section{References}

1 R.M. Weikle II, T.W. Crowe, T.W.; E.L. Kollberg, Inter. J. of High Speed Electronics \& Systems 13 (2003) 429

2 R. Kohler, A. Tredicucci, F. Beltram F, H.E. Beere, E.H. Linfield, A.G. Davies, D.A. Ritchie, Appl. Phys. Lett. 80 (2002) 1867

3 B.S. Williams, S. Kumar, H. Callebaut, Qing Hu, J.L. Reno, Appl. Phys. Lett. 83 (2003) 2124.

4 L. Esaki and R. Tsu, I.B.M. J. Res. Dev. 14 (1970) 61.

5 S.A Ktitorov, G.S. Simin, and Y. Sindalovski, Fiz. Tverd Tela 13 (1971) 2230 [Sov. Phys. Solid State 13 (1972) 1872].

6 P.G. Savvidis, B. Kolasa, G. Lee, and S.J. Allen, Phys. Rev. Lett., 92 (2004) 196802.

7 W. Shockley, Bell Syst. Tech. J., 33 (1954)799.

8 H. Kroemer, Proc. IEEE, 58 (1970) 1844.

9 S.M. Sze, High-Speed Semiconductor Devices WileyInterscience, 1990.

10 A.A Ignatov, V.I. Piskarev and V.I. Shashkin, Fiz. Tek. Pol., 19, (1985) 2183; [Sov. Phys. Semicond., 19 (1985) 1345].

11 B.J. Keay, S.J. Allen Jr, J. Galan, J.P. Kaminki, K.L. Campman, A.C. Gossard, U. Bhattacharya, M.J.W. Rodwell, Phys. Rev. Lett., 75 (1995) 4098.

12 K. Unterrainer, B.J. Keay, M.C. Wanke, S.J. Allen, D. Leonard, G Medeiros-Ribeiro, U. Bhattacharya, M.J.W. Rodwell, Phys. Rev. Lett., 76, (1996) 2973.

13 J.R. Tucker, IEEE J. Quantum Electron., QE-15 (1979) 1234; J.R. Tucker and M.J. Feldman, Rev. Mod. Phys., 57 (1985) 1055.

14 A. Wacker, Physics Reports, 357 (2002) 1. 